\title{
Philosophiques
}

\section{Alexis Klimov. Éloge de l'homme inutile. Québec, Éd. du Beffroi, 1983, 96 p. ; Diversions. Huit opérations poétiques pour une stratégie métaphysique. Québec, Éd. du Beffroi, 1983, 192 p. ; Veilleurs de nuit. Québec, Éd. du Beffroi, 1984, 86 p.}

\section{Jean-Claude Simard}

Volume 12, numéro 2, automne 1985

URI : https://id.erudit.org/iderudit/203296ar

DOI : https://doi.org/10.7202/203296ar

Aller au sommaire du numéro

Éditeur(s)

Société de philosophie du Québec

ISSN

0316-2923 (imprimé)

1492-1391 (numérique)

Découvrir la revue

Citer ce compte rendu

Simard, J.-C. (1985). Compte rendu de [Alexis Klimov. Éloge de l'homme inutile. Québec, Éd. du Beffroi, 1983, 96 p. ; Diversions. Huit opérations poétiques pour une stratégie métaphysique. Québec, Éd. du Beffroi, 1983, 192 p.; Veilleurs de nuit. Québec, Éd. du Beffroi, 1984, 86 p.] Philosophiques, 12(2), 445-451.

https://doi.org/10.7202/203296ar d'utilisation que vous pouvez consulter en ligne. 


\section{COMPTES RENDUS}

ALEXIS KLIMOV. Éloge de l'bomme inutile. Québec, Éd. du Beffroi, 1983, 96 p. ; Diversions. Huit opérations poétiques pour une stratégie métaphysique. Québec, Éd. du Beffroi, 1983, 192 p. ; Veilleurs de nuit. Québec, Éd. du Beffroi, 1984, $86 \mathrm{p}$.

\section{par Jean-Claude Simard}

En 1858, Gontcharov publiait le roman qui lui a ouvert les voies de la postérité : Oblomov. C'est l'histoire d'un propriétaire terrien, habitant SaintPétersbourg, qui cultive comme son bien le plus précieux un penchant naturel à la paresse. D'une aboulie chronique et d'une indécrottable apathie, ce personnage passe ses jours à s'incruster dans son meuble favori, un divan. Même l'amour d'Olga, dont on eût pu croire un moment qu'il aurait su le tirer de son état, se révèle en définitive insuffisant pour vaincre sa force d'inertie. Oblomov terminera ses jours dans la voie qu'il a choisie : faire corps avec son bien-aimé divan.

Ce personnage est devenu en Russie ${ }^{1}$ un type et a permis, grâce au célèbre critique Dobrolioubov, l'apparition d'un terme nouveau : l'oblomovisme. On a pu écrire à ce propos : "Oblomov est l' " homme de trop " type, issu d'une lignée qui remonte à Onéguine, mais il a aussi les vertus d'un cas-limite, facilement voué à transcender l'histoire ".$^{2}$

Ce que M. Klimov nous présente, dans ces trois ouvrages publiés presque coup sur coup, c'est une nouvelle version assez singulière de l'Homme en trop, de l'homme qui est, comme l'affirme le titre de l'un de ses ouvrages, " inutile ». Oblomov se caractérisait par un refus total de tout geste, de toute action pouvant mener à un résultat concret. Seul l'intéressait le monde de la rêverie improductive. Klimov nous propose le même rejet : le monde

1. Il est d'usage lorsqu'on parle de la culture historique de l'Union Soviétique d'employer le mot " russe ". Le terme n'est pas très heureux mais il faut bien reconnaître que nous n'en avons pas d'autre. Ne conformant à l'usage habituel, je l'utilise donc, tout en étant conscient qu'il souffre d'inflation sémantique caractérisée et qu'il en arrive à signifier, dans l'usage courant, l'essence du slavisme.

2. Bonamour, Jean, Le Roman Russe. Paris, P.U.F. (« Litt. modernes ", 16), 1978, p. 11. Voir encore dans le même sens Marie Maline (Précis de Littérature Russe, Bruxelles, Centre national pour l'étude des États de l'Est, 1964, p. 61) : "C'est une nouvelle variation dans la famille des «hommes de trop»". 
extérieur, celui qui est soumis au principe de réalité, est le règne de la " quotidienneté ", le domaine de l'objectivation. Mais à la différence d'Oblomov - et cette différence est capitale - Klimov propose en contrepartie une descente à l'intérieur de soi, une navigation sur les eaux de la mer intérieure, en somme, une aventure spirituelle. L'homme inutile, écrit-il, c'est celui qui « s'engage dans la nuit, dans la nuit de l'inconnu, plutôt que de s'adapter à une existence placée sous le signe de la quotidienneté. Pour l'homme inutile, exister n'est rien (. . .) vivre est tout ${ }^{3}$.

Cette affirmation donne le ton général des trois ouvrages. Mais avant de la décortiquer et d'en étudier les implications, présentons d'abord brièvement chacun de ceux-ci.

L'Éloge de l'Homme Inutile est le discours de réception de M. Klimov à la Société Royale du Canada. Il a été prononcé à l'Université du Québec à Trois-Rivières en mars 1983 et il est ici précédé d'une intéressante " présentation d'Alexis Klimov " par Clément Marchand, lequel trace un portrait assez réussi de l'auteur. Le second ouvrage, Diversions (dorénavant $D$.) est en fait un recueil d'articles, huit pour être exact, échelonnés de 1965 à 1981, qui avaient été publiés dans diverses revues peu connues ou peu accessibles et qu'on a cru bon de réunir en volume « parce que le grand public pourrait y trouver (. . .) matière à réflexion " ${ }^{4}$. Mentionnons immédiatement, puisque je n'aurai pas l'occasion d'y revenir, que l'une de ces huit études, intitulée "Leibniz et l'abbé de Saint-Pierre ", s'éloigne très nettement, autant par son contenu que par son caractère, des sept autres, comme aussi des deux plaquettes ici recensées. C'est la seule en effet qui puisse présenter un intérêt "scientifique ", ou pour les historiens de la philosophie ou pour les spécialistes de Leibniz. L'auteur y donne entre autres choses la traduction d'une assez longue lettre de Leibniz à l'abbé de Saint-Pierre, laquelle, à sa connaissance, "n'a jamais été présentée au lecteur français ". Il s'agit d'un commentaire poli, mais assez critique, du volumineux Projet de Paix Perpétuelle du bon abbé, dont on sait qu'il a inspiré le célèbre opuscule du même titre de Kant.

Le troisième et dernier ouvrage est la reprise d'une conférence sur Gabriel Marcel, prononcée lors de l'assemblée générale de l'Association internationale "Présence de Gabriel Marcel " tenue à Paris en octobre 1983. Le but en était simple : « rendre hommage à Louis Lavelle et Gabriel Marcel ${ }^{6}$ ", qu'il considère tous deux comme des "veilleurs de nuit " ${ }^{7}$, c'est-à-dire des pen-

3. Éloge de l'Homme Inutile (dorénavant E.), p. 56.

4. D., p. 9. L'affirmation semble exacte dans certains cas mais je doute par ailleurs que « le grand public " manifeste un enthousiasme délirant pour des études sur "Le Château de Villiers de L'Isle-Adam » ou sur «Boehme et la langue adamique ", respectivement les chapitres $\mathrm{V}$ et III . .

5. D., p. 61. Retrouvée dans les manuscrits de la bibliothèque Lénine de Moscou, elle paraissait en 1964 dans la revue soviétique Problèmes de Pbilosophie.

6. Veilleurs et Nuit (dorénavant V.), p. 21-22.

7. Le terme est emprunté, semble-t-il, à un essai de Lichtenberg. 
seurs ayant tenté cette aventure intérieure à laquelle il convie sans cesse ses lecteurs et lectrices. C'est cette parenté entre ces deux auteurs, surprenante à première vue, qui justifie sans doute l'inclusion, pp. 59 ss., d'un deuxième texte consacré à Louis Lavelle et lu au Colloque commémorant le centenaire de sa naissance, colloque tenu lors du Congrès Mondial de Philosophie à Montréal en 1983.

" Exister n'est rien, vivre est tout ».

Je rappelais tout à l'heure ce passage de l'Éloge de l'Homme Inutile en prétendant qu'il offrait un reflet assez fidèle du ton général des trois ouvrages. C'est que, pour Klimov, chacun, face à sa propre existence, doit opérer un choix, lequel présente une alternative claire : objectivité ou subjectivité, extériorité ou intériorité 8 . Il symbolise ce choix par la célèbre image de la croisée des chemins, illustrée à la Renaissance par Hercule devant opter pour le vice ou la vertu?. Pour Klimov, le choix est clair : " que voulez-vous : je fais partie, dit-il, de ces originaux qui n'hésitent pas à s'approprier cette parole de Soljénitsyne : 'Je suis non objectif et j'en suis fier»" $(V ., 18)$. Le choix opéré, on se retrouve devant le chemin vers soi-même, et l'on connaît, en s'engageant sur cette route, les affres de l'angoisse. Les divers systèmes, en particulier les systèmes philosophiques, ont pour fonction de masquer cette angoisse. Comprise correctement, elle est en fait le signe de la liberté totale, celle qu'ont connue entre autres les grands créateurs. Le trajet n'est pas sans danger et si le voyageur s'égare dans cette " nuit obscure » (St-Jean de la Croix), " plus rien, alors, ne peut le préserver des sables mouvants de la folie. Plus rien pour le détourner du suicide si, à force de côtoyer les abîmes, le vertige devenant insupportable, le désir d'en finir au plus vite rend même impossible de rebrousser chemin ". (D., 90) Ces dangers sont symbolisés par le tableau de " La chute d'Icare ", dont notre auteur fait une exégèse assez surprenante, mais qui est en tous points conforme à ses thèses principales. C'est selon lui, le symbòle de toute l'aventure humaine ${ }^{10}$. C'est ici le lieu de toucher un mot de la méthode employée par Klimov, dont l'interprétation de ce tableau est assez caractéristique. Il ne se préoccupe ni des intentions du peintre, ni de la place du tableau dans son œuvre (qui pourrait aider à en déterminer le sens réel), ni de l'éventuelle évolution

8. Ces deux termes qui reviennent sans cesse sous la plume de l'auteur ne sont jamais définis clairement et il faut, selon la méthode qui lui est chère, en circonscrire peu à peu le sens au fil du texte. Je ne crois pas trahir sa pensée en supposant qu'il faut les entendre en un sens très général, assez proche de ce que Jung proposait pour " introversion " et " extraversion ", i.e. deux directions opposées du regard et, souvent, deux modes de vie. À la limite, mêrne, deux modes d'être.

9. V., 44. Dürer en a donné une gravure célèbre. L'apologue, dû, semble-t-il à Prodicos, a été narré en détail par Xénophon (Mémorables, II, 1, 21-34) et est utilisé depuis les Stoïciens au moins, on le sait, pour symboliser un choix de vie fondamental.

10. E., 61. L'éditeur (?) a d'ailleurs choisi ce tableau pour orner la couverture de l'ouvrage. Mentionnons en passant l'exceptionnelle qualité de l'édition de ces trois ouvrages : précision des reproductions, caractères larges et lisibles, index des noms, index des personnages et des œuvres littéraires, etc. On rencontre assez rarement dans le milieu québecois du travail d'une telle valeur. 
artistique de l'auteur, ni de l'époque où il a été peint, non plus que de toute autre considération qui puisse permettre de poser ce qu'il est convenu d'appeler un jugement critique. Il en est de même, sans exception, de tous les auteurs dont il " commente " les textes ou les œuvres. L'idée qu'il puisse exister des interprétations exactes ou inexactes d'une œuvre et que des critères précis rendent possible une telle détermination ne semble jamais effleurer son esprit. Au premier abord, on trouve cette procédure tendancieuse, pour ne pas dire carrément extravagante. Jusqu'à ce qu'on saisisse (c'est du moins ce qu'il faut, je l'espère, comprendre) qu'il y a là, pour l'auteur, une façon de revendiquer jusqu'au bout sa subjectivité. En d'autres termes, ces œuvres, poèmes, textes ou tableaux, ne sont pas pour lui des documents, mais des témoignages. Il appliquerait en somme, indépendamment de tout autre souci, le mot de Molière : "Je prends mon bien où je le trouve ".

Sur sa lancée, le voyageur aboutit à des difficultés qui se muent bientôt en contradictions insurmontables. Est nié alors le principe de non-contradiction sur lequel repose toute entreprise rationnelle. Il faut dorénavant " assumer les conséquences existentielles de la destruction " $(V ., 41)$ de ce principe : l'intellect et la raison sont facteurs d'objectivation et donc de mort. Selon lui, la raison, " appesantie par ses impuretés (est) positivement réfractaire à toute forme et à tout mode d'élévation spirituelle » $(V ., 46)$.

Poussées à leur limite, ces contradictions détruisent le langage qui repose sur le principe de non-contradiction. Seuls les symboles, dont l'auteur fait un usage étendu, traduisent alors l'expérience vécue et l'on aboutit au silence. Cet état est ouverture sur le Sacré, la plus haute expression du spirituel, et celle où l'on atteint, selon le mot célèbre de Rudolf Otto, la « coïncidentia oppositorum » (D., 20) ou encore, comme l'a dit Breton, ce "point de l'esprit où les choses cessent d'être perçues contradictoirement » (D., 106). Ce point, les philosophes l'auraient, selon l'auteur, nommé indifféremment «Dieu, Logos, Ungrund, Absolu, Totalité, Un, etc. " (D., 20). C'est encore ce même point que rejoignent les mystiques. Ainsi la religion, comprise de manière non-exotérique, i.e. vécue à l'extérieur de l'institution ecclésiale, des dogmés et des systèmes théologiques, équivaut à la philosophie comprise comme aventure existentielle, puisque les deux sont, au sens large, spirituelles. Il faut, selon lui, "reconnaître l'impossibilité de définir la philosophie » $(D ., 22)$ qui n'est, au fond, qu'une " tentative de cerner, d'approcher l'inexprimable " $"$.

Par ailleurs, toute vie, à condition qu'elle se déploie dans ce domaine qui tend vers l'intériorité, aboutit au même résultat. Toutes les entreprises humaines qui ne font pas un usage privilégié de la Raison - en conséquence il faut apparemment exclure à tout le moins les sciences et les techniques - sont donc parentes et, bien plus, visent le même but. Ainsi, l'art et la littérature disent la même chose que la religion ou la philosophie (bien

11. D., 24. Voir à ce propos tout le chapitre intitulé «Faut-il succomber à la tentation de définir la philosophie de la religion?", pp. 13-27. 
comprises). Lorsqu'on surmonte les épreuves de la vie, affirme-t-il, on s'approche « de cette coïncidence des opposés dans laquelle poètes, artistes, métaphysiciens ${ }^{12}$ et mystiques ont vu le fondement de tout ce qui est $\gg(D$., 140). Il devient alors loisible d'affirmer que Berdiaeff et Picasso se rejoignent (D., chap. VII), ou que les cubistes sont philosophes ou que Villiers de l'IsleAdam et Keyserling sont métaphysiciens, etc.

Philosophe, poète et mystique, ces termes ne (servent) quà distinguer des modalités - notamment dans le domaine de l'expression et du langage - renvoyant à une même démarche fondamentale : la quête de l'absolu, de la totalité, de la plénitude. ${ }^{13}$

Subjectivité, existence, objectivation : on reconnaît dans les prises de position globales de Klimov le vocabulaire et le schéma général de l'existentialisme. En effet, là aussi, l'existence humaine est la seule réalité substantielle. Il importe d'échapper au règne du " on " (Heidegger), de l'« objectivation » (Berdiaeff), de l'« avoir » (Marcel). Cela n'est possible que grâce à un engagement, une prise en charge de sa liberté. Vécue dans ses ultimes conséquences, ce choix fondamental mène à l'authenticité. Alors, selon la célèbre formule de Kierkegaard, la subjectivité est la vérité.

L'existentialisme en général pose des difficultés auxquelles ses défenseurs ont parfois cherché à remédier. L'existentialisme assez particulier de Klimov n'échappe pas à ces problèmes. Soulignons-en quelques-uns.

Que l'existence individuelle, très individuelle soit la réalité ultime et son exploration l'expression de la vérité, cette thèse ne peut être rejetée a priori. Il s'agit là d'une option philosophique de base. Une telle prise de position est à mon sens assez limitative (elle suppose le caractère substantiel du soi, l'indépendance de la subjectivité, toutes choses qui ne sont pas, je l'indiquerai à l'instant, évidentes), mais elle est défendable. L'ennui est que l'auteur ne tente jamais de le faire. Par exemple, il se pourrait que l'existence humaine soit indissolublement duelle parce que résultant d'une interaction entre intériorité et extériorité (pour reprendre la terminologie de l'auteur). Privilégier l'intériorité serait alors un choix, réduire l'extériorité à l'objectivation serait manifestement une erreur. Comme l'affirme Mounier, "le rapport de la personne à la nature n'est donc pas un rapport de pure extériorité, mais un rapport dialectique d'échange et d'ascension ${ }^{14}$.

Autre objection, plus particulière à l'existentialisme de l'auteur : l'art, la religion et la philosophie se rejoignent, croit-il, parce que toutes trois supposent un vécu intense. Soit. Mais alors pourquoi écarter, par exemple, Darwin ou Einstein ? La science ne suppose-t-elle pas une ascèse tout aussi rigoureuse que celles de l'art ou de la philosophie ? Le critère de démarcation

12. Il ne faut pas entendre par là la métaphysique au sens courant puisque, pour Klimov, Aristote a apporté à l'Occident "la première grande cuvre anti-métaphysique " $(V$., 36) ! Métaphysique veut dire ici compréhension spirituelle de la vie.

13. D., 37.

14. Le Personnalisme, Paris, P.U.F. ("Que sais-je ?", 395), 1969, p. 30. 
serait-il l'« extériorité » plutôt que l'intensité du vécu ? Il faudrait alors écarter aussi l'artiste (que serait un artiste sans extériorité, i.e. sans œuvre ?) de cette constellation de vies authentiques...

On pourrait multiplier longuement ce genre d'objections latérales, mais ce serait finalement assez fastidieux. Allons plutôt directement à l'éssentiel.

La subjectivité et sa forme particulière revendiquée ici, l'intériorité, ont été largement critiquées depuis trente ans. Sans même faire appel au domaine anglo-saxon et pour rester dans le cadre français, on songe par exemple à toute l'entreprise dite structuraliste, particulièrement aux travaux de Lacan, Foucault, Barthes et Derrida. On peut résumer leur critique de la subjectivité en la subsumant sous trois axes fondamentaux de questionnement. Se pourraitil que la transparence du sujet - et donc la possibilité d'une égalité de soi à soi, d'une connaissance de soi sur laquelle repose toute la démarche de pensée de Klimov - soit un mythe, né pour des raisons historiques et entretenu pour les mêmes raisons ? Se pourrait-il encore que, plus fondamentalement, l'intériorité du sujet elle-même soit illusoire parce qu'elle suppose que le langage ne serait que l'expression de la substance-sujet alors que la réalité pourrait bien être inverse ? Se pourrait-il enfin, et radicalement, que le sujet lui-même ne soit au fond qu'un " effet de langage ", un " pli de la représentation » et que, comme le suggère Barthes ${ }^{15}$, le langage soit lui-même le sujet?

Après la vague existentialiste des années 40 et 50 , ces questions méritaient d'être posées. Les réponses affirmatives des divers structuralistes, habituellement sans équivoque, pourraient être partiellement erronées, mais encore faudrait-il en faire la démonstration. Or, si $\mathrm{M}$. Klimov est conscient des difficultés que ces diverses réponses posent pour son entreprise philosophique, s'il a jamais tenté d'y réfléchir, rien en tout cas n'en transparaît dans les trois textes que $j$ 'ai sous la main. Les lisant, on a l'impression très nette que la philosophie des trente dernières années n'a pas existé. Si l'on croit à la nécessité pour une pensée vigoureuse d'affronter les problèmes philosophiques de l'heure et d'élaborer sa tâche de pensée à l'intérieur du champ de questionnement ouvert par l'époque, alors on sera très surpris par ces ouvrages qui enjambent assez cavalièrement ces exigences. Si par contre, on croit plutôt que certaines problématiques sont inévitables et qu'elles récoltent leur intemporalité du fait qu'elles ne sauraient s'abaisser à traiter les problèmes de l'heure, alors on se réjouira sans doute de ce que l'auteur agisse comme il le fait. Pour ce lecteur-ci, qui penche plutôt vers le premier terme de l'alternative, on obtient ainsi une philosophie qui, malgré sa volonté de s'inscrire au creux même de l'existence, demeure bien désincarnée. Si l'intemporalité est à ce prix, mieux vaut l'oublier. Pour l'auteur, qui favorise à n'en pas douter l'autre partie de l'alternative, c'est sans doute une forme de fierté que cette sorte particulière d'intemporalité. Et sans doute est-ce là aussi un des

15. Critique et Vérité, Paris, Seuil, 1966, p. 70. 
sens ultimes de son entreprise philosophique, comme il le dit dans Veilleurs de Nuit (p. 33) :

Or, l'essentiel ne réside-t-il pas dans cette façon de rechercher la Sagesse qui consiste, pour reprendre les termes admirables de Gabriel Marcel, "à s'aventurer $(.$.$) sur les chemins qui conduisent (. . .) hors de notre$ temps "?

Nous avions commencé cette recension en situant le travail de Klimov à l'intérieur d'une tradition russe, l'oblomovisme, revendication de l'inutilité comme principe régulateur de l'existence. Nous avons vu en cours de route comment l'auteur lui infusait une coloration toute particulière. Reste malgré tout une question, fondamentale : la philosophie doit-elle, à notre époque, être encore cette révolte formelle contre l'objectivation, splendide et inutile ? En d'autres termes un oblomovisme, tout spirituel fût-il, a-t-il aujourd'hui un sens ? La lecture de ces trois ouvrages d'Alexis Klimov oblige à poser la question. Elle ne permet pas d'y répondre.

Département de philosophie

Collège de Rimouski 